\title{
BOUNDS FOR INTEGRAL SOLUTIONS OF DIAGONAL CUBIC EQUATIONS
}

BY

\author{
KA-HIN LEUNG ${ }^{1}$
}

\begin{abstract}
It was proved by Davenport [3] that for the nonzero integral $\lambda_{i}$ the equation $\lambda_{1} x_{1}^{3}+\cdots+\lambda_{8} x_{8}^{3}=0$ always has a nontrivial integral solution. In this paper, we investigate the bounds of nontrivial integral solutions in terms of $\lambda_{1}, \ldots, \lambda_{8}$.
\end{abstract}

1. Introduction. Pitman and Ridout [9] proved that for every $\theta>0$, there exists a constant $c_{\theta}$ with the following property. If $\lambda_{1}, \ldots, \lambda_{9}$ are nonzero integers, then the equation

$$
\lambda_{1} x_{1}^{3}+\cdots+\lambda_{9} x_{9}^{3}=0
$$

has a solution in nonzero integers $x_{1}, \ldots, x_{9}$, such that

$$
\left|\lambda_{1} x_{1}^{3}\right|+\cdots+\left|\lambda_{9} x_{9}^{3}\right|<c_{\theta}\left|\lambda_{1} \cdots \lambda_{9}\right|^{(3 / 2)+\theta} .
$$

They conjectured [9] that it should be possible to obtain bounds for integral solutions of the equation

$$
\lambda_{1} x_{1}^{3}+\cdots+\lambda_{8} x_{8}^{3}=0
$$

where $\lambda_{1}, \ldots, \lambda_{8}$ are nonzero integers.

However, their method cannot be directly extended. In this paper we shall use I. Danicic's [7] idea and improve Davenport and Roth's [6] results to overcome the difficulties and prove the following

THEOREM 1. For every $\theta>0$, there exists a constant $c_{\theta}$, depending on $\theta$ only, with the following property. If $\lambda_{1}, \ldots, \lambda_{8}$ are nonzero integers, and not all of the same sign, then (2) has a solution in positive integers $x_{1}, \ldots, x_{8}$ such that

$$
\left|\lambda_{1} x_{1}^{3}\right|+\cdots+\left|\lambda_{8} x_{8}^{3}\right|<c_{\theta}\left|\lambda_{1} \cdots \lambda_{8}\right|^{(35 / 8)+\theta} .
$$

2. Notation and general lemmas. Let $\lambda_{i}(i=1, \ldots, 8)$ be given nonzero integers such that $P \geqslant\left|\lambda_{i}\right|$ for all $i$. We write

$$
\Pi=\prod_{i=1}^{8}\left|\lambda_{i}\right|
$$

and define

$$
S_{i}(\alpha)=\sum_{x_{i}} e\left(\lambda_{i} x_{i}^{3} \alpha\right), \quad i=1, \ldots, 8,
$$

Received by the editors February 24, 1982.

1980 Mathematics Subject Classification. Primary 10B10.

${ }^{1}$ Thanks are due to Dr. M. C. Liu for his valuable advice and encouragement, and to the Swire group for the Swire Scholarship offered. 
where $e(y)=\exp (2 \pi y i)$ and $x_{i}$ runs through all integral values in the range

$$
\begin{gathered}
P \leqslant\left|\lambda_{i}\right|^{1 / 3} x_{i} \leqslant 2 P, \quad i=1, \ldots, 4, \\
P^{4 / 5} \leqslant\left|\lambda_{j}\right|^{1 / 3} x_{j} \leqslant 2 P^{4 / 5}, \quad j=5, \ldots, 8 .
\end{gathered}
$$

Throughout the paper, the letters $a, q, a_{i}, q_{i}$ always denote integers. In the following lemmas, $\delta$ denotes a fixed small positive number and $\varepsilon$ denotes an arbitrary small positive number, not the same throughout. The constants implied by the notation 0 , $\ll, \gg$ are always independent of the $\lambda_{i}$ and of $P$, and without loss of generality, we assume the constants are greater than or equal to 1 . In this section they depend only on $\delta$ and $\varepsilon$; in later sections they will depend only on $\theta, \delta$ and $\varepsilon$ and so ultimately on $\theta$, since $\delta$ and $\varepsilon$ will be determined by $\theta$.

LEMMA 2.1. (i) If $p$ is prime and $(a, p)=1$, then

$$
S(a, p) \leqslant 2 P^{1 / 2} \text {. }
$$

(ii) If $(a, q)=1$, then $S(a, q) \ll q^{2 / 3}$ where $S(a, k)=\sum_{x=1}^{k} e\left(a x^{3} / k\right)$.

Proof. Both (i) and (ii) are particular cases of Lemmas 12 and 15, respectively, of [4].

LEMMA 2.2. If $|\beta| \leqslant \frac{1}{2}$, then

$$
I(\beta)=\sum_{m=P^{3}}^{8 P^{3}} \frac{1}{3} m^{-2 / 3} e(\beta m) \ll P \min \left(1, P^{-3}|\beta|^{-1}\right)
$$

and

$$
I^{\prime}(\beta)=\sum_{m=P^{12 / 5}}^{8 P^{12 / 5}} \frac{1}{3} m^{-2 / 3} e(\beta m) \ll P^{4 / 5} \min \left(1, P^{-12 / 5}|\beta|^{-1}\right) .
$$

Proof. See [1, Lemma 3].

Now for all $\alpha \in[0,1]=I, \lambda_{i} \alpha$ can be represented in the form

(4)

$$
\left\{\begin{array}{c}
\lambda_{i} \alpha=\left(a_{i} / q_{i}\right)+\beta_{i} \quad \text { where }\left(a_{i}, q_{i}\right)=1, \\
0<q_{i} \leqslant\left(\left|\lambda_{i}\right|^{-1 / 3} P\right)^{2+\delta}, \quad\left|\beta_{i}\right| \leqslant q_{i}^{-1}\left(\left|\lambda_{i}\right|^{-1 / 3} P\right)^{-2-\delta}, \quad i=1, \ldots, 4, \\
0<q_{i} \leqslant\left(\left|\lambda_{i}\right|^{-1 / 3} P^{4 / 5}\right)^{2+\delta}, \quad\left|\beta_{i}\right| \leqslant q_{i}^{-1}\left(\left|\lambda_{i}\right|^{-1 / 3} P^{4 / 5}\right)^{-2-\delta}, \quad i=5, \ldots, 8 .
\end{array}\right.
$$

LEMMA 2.3. Suppose that $\lambda_{i} \alpha$ is in the form of (4). Then:

(i) If $1 \leqslant i \leqslant 4$,

$$
\begin{aligned}
S_{i}(\alpha) & =\left|\lambda_{i}\right|^{-1 / 3} q_{i}^{-1} S\left(a_{i}, q_{i}\right) I\left( \pm \beta_{i} / \lambda_{i}\right)+O\left(q_{i}^{2 / 3+\varepsilon}\right) \\
& \ll\left|\lambda_{i}\right|^{-1 / 3} q_{i}^{-1 / 3} P \min \left(1, P^{-3}\left|\lambda_{i} / \beta_{i}\right|\right) .
\end{aligned}
$$

(ii) If $5 \leqslant i \leqslant 8$,

$$
\begin{aligned}
S_{i}(\alpha) & =\left|\lambda_{i}\right|^{-1 / 3} q_{i}^{-1} S\left(a_{i}, q_{i}\right) I^{\prime}\left( \pm \beta_{i} / \lambda_{i}\right)+O\left(q_{i}^{2 / 3+\varepsilon}\right) \\
& \ll\left|\lambda_{i}\right|^{-1 / 3} q_{i}^{-1 / 3} P^{4 / 5} \min \left(1, P^{-12 / 5}\left|\lambda_{i} / \beta_{i}\right|\right)
\end{aligned}
$$

where \pm is the sign of $\lambda_{i}$. 
Proof. This is essentially the same as [1, Lemmas 7-10].

Lemma 2.4. Suppose that $\lambda_{i} \alpha$ satisfies (4) and, in particular,

$$
\begin{gathered}
\left(\left|\lambda_{i}\right|^{-1 / 3} P\right)^{-1-\delta}<q_{i} \leqslant\left(\left|\lambda_{i}\right|^{-1 / 3} P\right)^{2+\delta} \quad \text { if } 1 \leqslant i \leqslant 4 \\
\left(\left|\lambda_{i}\right|^{-1 / 3} P^{4 / 5}\right)^{1-\delta}<q_{i} \leqslant\left(\left|\lambda_{i}\right|^{-1 / 3} P^{4 / 5}\right)^{2+\delta} \quad \text { if } 5 \leqslant i \leqslant 8 .
\end{gathered}
$$

Then

$$
\begin{aligned}
& S_{i}(\alpha) \ll\left(\left|\lambda_{i}\right|^{-1 / 3} P\right)^{3 / 4+\delta} \quad \text { if } 1 \leqslant i \leqslant 4, \\
& S_{i}(\alpha) \ll\left(\left|\lambda_{i}\right|^{-1 / 3} P^{4 / 5}\right)^{3 / 4+\delta} \quad \text { if } 5 \leqslant i \leqslant 8 .
\end{aligned}
$$

Proof. See [1, Lemma 13].

Before we proceed, we rearrange $\lambda_{i}$ so that

$$
\left|\lambda_{1}\right| \geqslant\left|\lambda_{2}\right| \geqslant\left|\lambda_{j}\right|, \quad j=3, \ldots, 8,
$$

and if $\lambda_{1} \lambda_{2}>0$, choose $\lambda_{3}$ s.t. $\lambda_{1} \lambda_{3}<0$ and define, for any $g \geqslant 0, h \geqslant 0$,

$$
K_{i j}(g, h)=\int_{I}\left|S_{i}(\alpha)\right|^{g}\left|S_{j}(\alpha)\right|^{h} d \alpha
$$

for $i=1, \ldots, 4, j=5, \ldots, 8$. In proving the following lemmas, (6) is assumed.

LEMMA 2.5 .

$$
\begin{aligned}
& K_{i j}(0,4) \ll\left(\left|\lambda_{j}\right|^{-1 / 3} P^{4 / 5}\right)^{2+\varepsilon} . \\
& K_{i j}(0,8) \ll\left(\left|\lambda_{j}\right|^{-1 / 3} P^{4 / 5}\right)^{5+\varepsilon} .
\end{aligned}
$$

Proof. See [9, Lemma 5].

LEMMA 2.6. Let $N^{\prime}(m)$ be the number of solutions of $x^{3}+y^{3}-x^{\prime 3}-y^{\prime 3} \equiv 0$ $\bmod m$, where $1 \leqslant x, y, x^{\prime}, y^{\prime} \leqslant m$. Then $N^{\prime}(m) \ll m^{7 / 2+\varepsilon}$.

PROOF.

$$
\begin{aligned}
N^{\prime}(m) & =\int_{I}\left|\sum_{x=1}^{m} e\left(\alpha x^{3}\right)\right|^{4} \sum_{x=-2 m^{2}}^{2 m^{2}} e(m x \alpha) d \alpha \\
& \left.\left.\ll\left|\int_{I}\right| \sum_{x=1}^{m} e\left(\alpha x^{3}\right)\right|^{8} d \alpha \int_{I}\left|\sum_{x=-2 m^{2}}^{2 m^{2}} e(m x \alpha)\right|^{2} d \alpha\right)^{1 / 2} \\
& \ll m^{7 / 2+\varepsilon} \quad \text { by Hua's inequality. }
\end{aligned}
$$

LEMMA 2.7.

$$
\begin{aligned}
& K_{i j}(2,4) \ll\left|\lambda_{i}\right|^{-1 / 3}\left|\lambda_{j}\right|^{-2 / 3} P^{13 / 5+\varepsilon} . \\
& K_{i j}(2,6) \ll\left|\lambda_{i}\right|^{-1 / 3}\left|\lambda_{j}\right|^{-7 / 6} P^{19 / 5+\varepsilon} .
\end{aligned}
$$

Proof. It is essentially the same as [6, Lemmas 5 and 6] for $\left|\lambda_{i}\right| \leqslant\left|\lambda_{j}\right|$. Since we may have $\left|\lambda_{i}\right| \geqslant\left|\lambda_{j}\right|$, we need Lemma 2.6 to improve the result. We omit (ii) and 
prove (i) only. The integral is equal to the number of solutions

$$
\lambda_{i}\left(x^{3}-x^{\prime 3}\right)+\lambda_{j}\left(y^{3}+z^{3}-y^{\prime 3}-z^{\prime 3}\right)=0
$$

in integers satisfying

$$
P \leqslant\left|\lambda_{i}\right|^{1 / 3} x, \quad\left|\lambda_{i}\right|^{1 / 3} x^{\prime} \leqslant 2 P, \quad P^{4 / 5} \leqslant\left|\lambda_{j}\right|^{1 / 3}\left(y, z, y^{\prime}, z^{\prime}\right) \leqslant 2 P^{4 / 5} .
$$

For these solutions with $x=x^{\prime}$, the number of pairs of $y, z, y^{\prime}, z^{\prime}$ satisfying $(8) \ll\left(\left|\lambda_{j}\right|^{-1 / 3} P^{4 / 5}\right)^{2+\varepsilon}$. The number of this kind of solution is therefore

$$
\ll\left|\lambda_{i}\right|^{-1 / 3}\left|\lambda_{j}\right|^{-2 / 3} P^{13 / 5+\varepsilon} \text {. }
$$

Now we estimate the number $N$ of solutions with $x^{\prime}>x$. Let $x^{\prime}=x+t$. (8) becomes

$$
\lambda_{i}\left(3 x^{2} t+3 x t^{2}+t^{3}\right)-\lambda_{j}\left(y^{3}+z^{3}-y^{\prime 3}-z^{\prime 3}\right)=0,
$$

and we observe that $0<t \leqslant\left|\lambda_{i}\right|^{-1 / 3} P^{2 / 5}$. Let $N\left(t, y^{\prime}, z^{\prime}\right)$ denote the number of solutions for prescribed values of $t, y^{\prime}, z^{\prime}$. Then

$$
N=\sum_{t, y^{\prime}, z^{\prime}} N\left(t, y^{\prime}, z^{\prime}\right) \leqslant\left(\sum_{t, y^{\prime}, z^{\prime}} 1\right)^{1 / 2}\left(\sum_{t, y^{\prime}, z^{\prime}} N^{2}\left(t, y^{\prime}, z^{\prime}\right)\right)^{1 / 2}=\left(N_{1}\right)^{1 / 2}\left(N_{2}\right)^{1 / 2}
$$

say. It is obvious that $N_{1} \ll\left|\lambda_{i}\right|^{-1 / 3}\left|\lambda_{j}\right|^{-2 / 3} P^{2}$. Also $N_{2}$ represents the number of solutions of

$$
\begin{aligned}
& \lambda_{i}\left(3 x_{1}^{2} t+3 x_{1} t^{2}+t^{3}\right)-\lambda_{j}\left(y_{1}^{3}+z_{1}^{3}-y^{\prime 3}-z^{\prime 3}\right)=0, \\
& \lambda_{i}\left(3 x_{2}^{2} t+3 x_{2} t^{2}+t^{3}\right)-\lambda_{j}\left(y_{2}^{3}+z_{2}^{3}-y^{\prime 3}-z^{\prime 3}\right)=0 .
\end{aligned}
$$

The number of solutions of these simultaneous equations with $x_{1}=x_{2}$ is $\ll P^{\varepsilon} N$. With regard to the solution for $x_{1} \neq x_{2}$, that would imply

$$
3 \lambda_{i} t\left(x_{1}-x_{2}\right)\left(x_{1}+x_{2}+t\right)=\lambda_{j}\left(y_{1}^{3}+z_{1}^{3}-y_{2}^{3}-z_{2}^{3}\right)
$$

for values of $y_{1}, z_{1}, y_{2}, z_{2}$ such that the right-hand side is a multiple of $\lambda_{i}$. We can determine the values of $x_{1}, x_{2}, t$ with $p^{\varepsilon}$ possibilities, and the values of $y^{\prime}, z^{\prime}$ are then determined by either of the two equations with $p^{\varepsilon}$ possibilities. Thus the number of solutions with $x_{1} \neq x_{2}$ is $\ll\left(\left|\lambda_{j}\right|^{-1 / 3} P^{4 / 5}\right)^{4} m^{-4} N^{\prime}(m) P^{\varepsilon}$, where $m=\left|\lambda_{i} /\left(\lambda_{i}, \lambda_{j}\right)\right|$ and $N^{\prime}(m)$ is defined as in Lemma 2.6. Hence

$$
\ll\left|\lambda_{j}\right|^{-4 / 3} P^{16 / 5}\left(\left|\lambda_{i}\right| /\left|\lambda_{j}\right|\right)^{-1 / 3+\varepsilon}=\left|\lambda_{i}\right|^{-1 / 3}\left|\lambda_{j}\right|^{-1} P^{16 / 5+\varepsilon} .
$$

By a similar argument applied to (9),

$$
N \ll\left|\lambda_{i}\right|^{-1 / 3}\left|\lambda_{j}\right|^{-1} P^{16 / 5+\varepsilon} .
$$

It follows that $N_{2} \ll\left|\lambda_{i}\right|^{-1 / 3}\left|\lambda_{j}\right|^{-1} P^{16 / 5+\varepsilon}$, giving the desired result

$$
N \ll\left|\lambda_{i}\right|^{-1 / 3}\left|\lambda_{j}\right|^{-2 / 3} P^{13 / 5+\varepsilon} .
$$

$x^{\prime}<x$ is similar.

LeMmA 2.8. Let $A_{i}=\left\{\alpha \in I: S_{i}(\alpha) \gg\left|\lambda_{i}\right|^{-1 / 4} P^{3 / 4+2 \delta}\right\}, i=1, \ldots, 4$. Then:

$$
\int_{A_{i}}\left|S_{i}(\alpha)\right|^{6} d \alpha \ll\left|\lambda_{i}\right|^{-1} P^{3+\varepsilon} .
$$


(ii)

$$
\int_{A_{i}}\left|S_{i}(\alpha)\right|^{5} d \alpha \ll\left|\lambda_{i}\right|^{-3 / 4} P^{9 / 4-2 \delta+\varepsilon} .
$$

Proof. (i) For every $\alpha$ in $A_{i}$, we determine $a_{i}, q_{i}$ such that (4) is satisfied, so by Lemma 2.4, $0<q_{i} \leqslant\left(\left|\lambda_{i}\right|^{-1 / 3} P\right)^{1-\delta}$ and, by Lemma 2.3,

$$
\left|\lambda_{i}\right|^{-1 / 4} P^{3 / 4+2 \delta} \ll\left|S_{i}(\alpha)\right| \ll q_{i}^{-1 / 3}\left|\lambda_{i}\right|^{-1 / 3} P \min \left(1, P^{-3}\left|\lambda_{i} / \beta_{i}\right|\right)
$$

which imply

$$
0<q_{i} \ll\left|\lambda_{i}\right|^{-1 / 4} P^{3 / 4-6 \delta}, \quad\left|\beta_{i} / \lambda_{i}\right| \ll q_{i}^{-1 / 3}\left|\lambda_{i}\right|^{-1 / 12} P^{-11 / 4-2 \delta} .
$$

Let $F_{a_{i}, q_{i}}$ denote the interval of values of $\alpha$ satisfying (4) and (10). Since $A_{i} \subset \cup F_{a_{i}, q_{i}}$, where $q_{i}$ satisfies (10) and $\left|a_{i}\right| \leqslant\left|\lambda_{i} q_{i}\right|$,

$$
\int_{A_{i}}\left|S_{i}(\alpha)\right|^{6} d \alpha \ll \sum_{q_{i}} \sum_{a_{i}} \int_{F_{a_{i}, q_{i}}}\left|S_{i}(\alpha)\right|^{6} d \alpha
$$

where the summations $a_{i}, q_{i}$ are as before.

$$
\begin{aligned}
\int_{F_{a_{i}, q_{i}}}\left|S_{i}(\alpha)\right|^{6} d \alpha & \ll \int_{0}^{P^{-3}\left|\lambda_{i}\right|}\left|\lambda_{i}\right|^{-3} q_{i}^{-2} P^{6} d \beta_{i}+\int_{P^{-3}\left|\lambda_{i}\right|}^{\infty} P^{-12}\left|\lambda_{i}\right|^{3} q_{i}^{-2} \beta_{i}^{-6} d \beta_{i} \\
& \ll\left|\lambda_{i}\right|^{-2} q_{i}^{-2} P^{3} .
\end{aligned}
$$

So

(ii) follows easily from (i).

$$
\int_{A_{i}}\left|S_{i}(\alpha)\right|^{6} d \alpha \ll \sum_{q_{i}} \sum_{a_{i}}\left|\lambda_{i}\right|^{-2} q_{i}^{-2} P^{3} \ll\left|\lambda_{i}\right|^{-1} P^{3+\varepsilon}
$$

Lemma 2.9. Assume that $P>\Pi^{35 / 24}$. Then:

(i) For $i=1, \ldots, 4, j=5, \ldots, 8$,

$$
\begin{aligned}
& \int_{A_{i}}\left|S_{i}(\alpha)\right|^{3}\left|S_{j}(\alpha)\right|^{4} d \alpha \ll\left|\lambda_{i}\right|^{-9 / 16}\left|\lambda_{j}\right|^{-7 / 9} P^{67 / 20+2 \delta+\varepsilon} \quad \text { if } i=1, \\
& \ll\left|\lambda_{i}\right|^{-2 / 3}\left|\lambda_{j}\right|^{-7 / 9} P^{67 / 20+2 \delta+\varepsilon} \quad \text { if } i \neq 1 .
\end{aligned}
$$

(ii)

$$
K_{i j}(3,4) \ll\left|\lambda_{i}\right|^{-7 / 12}\left|\lambda_{j}\right|^{-2 / 3} P^{67 / 20+2 \delta+\varepsilon}, \quad i=2,3,4, j=5, \ldots, 8 .
$$

ProOf. (i)

$$
\begin{aligned}
\int_{A_{i}}\left|S_{i}(\alpha)\right|^{3}\left|S_{j}(\alpha)\right|^{4} d \alpha & \ll K_{i j}(2,6)^{2 / 3}\left(\int_{A_{i}}\left|S_{i}(\alpha)\right|^{5} d \alpha\right)^{j / 3} \\
& \ll\left|\lambda_{i}\right|^{-17 / 36}\left|\lambda_{j}\right|^{-7 / 9} P^{197 / 60+\varepsilon}
\end{aligned}
$$

Using $P>\Pi^{35 / 24}$, which implies $P>\left|\lambda_{1}\right|^{35 / 24}$ and $P>\left|\lambda_{i}\right|^{35 / 12}$ if $i \neq 1$, (i) follows.

(ii)

$$
\begin{aligned}
K_{i j}(3,4) & \ll \int_{A_{i}}\left|S_{i}(\alpha)\right|^{3}\left|S_{j}(\alpha)\right|^{4} d \alpha+\int_{I \backslash A_{i}}\left|S_{i}(\alpha)\right|^{3}\left|S_{j}(\alpha)\right|^{4} d \alpha \\
& \ll\left|\lambda_{i}\right|^{-7 / 12}\left|\lambda_{j}\right|^{-2 / 3} P^{67 / 20+2 \delta+\varepsilon}+\left|\lambda_{i}\right|^{-1 / 4} P^{3 / 4+2 \delta} K_{i j}(2,4) .
\end{aligned}
$$

By (i) and Lemma 2.7(i), (ii) follows. 
3. Minor ares for the proof of Theorem 1. We let $\Re(P)$ be the number of integral solutions of (2) which lie in the 'box' determined by (3). We shall show that for a given positive number $\theta$, there exists a positive constant $D_{\theta}$, independent of $\lambda_{i}$, such that if $P^{1-\theta}>D_{\theta} \Pi^{35 / 24}$, then $\Re(P)>0$. So we may take $P$ such that $D_{\theta} \Pi^{35 / 24}<$ $P^{1-\theta}<2 D_{\theta} \Pi^{35 / 24}$, which will imply there exists a solution of $(2)$ in nonzero integers with

$$
\sum_{i}\left|\lambda_{i} x_{i}^{3}\right|<8\left(2 D_{\theta} \Pi^{35 / 24}\right)^{3 /(1-\theta)}<K_{\theta} \Pi^{35 / 8(1-\theta)},
$$

where $K_{\theta}$ depends on $\theta$ only, so for every $\phi>0$, we can find $0<\theta<1$ such that $35 / 8(1-\theta)<35 / 8+\phi$, and hence Theorem 1 follows.

We now use

$$
\Re(P)=\int_{I} V(\alpha) d \alpha, \quad \text { where } V(\alpha)=\prod_{i=1}^{8} S_{i}(\alpha)
$$

and estimate $\mathscr{N}(P)$ will be of the form $c \Pi^{-1 / 3-\varepsilon} P^{21 / 5}$ where $c>0$, and the error terms are substantially smaller than $\Pi^{-1 / 3} P^{21 / 5}$ provided $P$ is substantially larger than $\Pi^{35 / 24}$.

By Dirichlet's theorem on diophantine approximation, for each $\alpha$ in $I$ and $i$, there exists a rational approximation $a_{i} / q_{i}$ to $\lambda_{i} \alpha$ such that (4) holds. Let $S=\cup_{i=1}^{8} B_{i}$ where

$$
\begin{array}{ll}
B_{i}=\left\{\alpha \in I: S_{i}(\alpha) \ll\left|\lambda_{i}\right|^{-1 / 4} P^{3 / 4+2 \delta}\right\}, & 1 \leqslant i \leqslant 4, \\
B_{j}=\left\{\alpha \in I: S_{j}(\alpha) \ll\left|\lambda_{j}\right|^{-1 / 4} P^{3 / 5+2 \delta}\right\}, & 5 \leqslant j \leqslant 8 .
\end{array}
$$

LEMMA 3.1. $\int_{S}|V(\alpha)| d \alpha \ll \Pi^{-3 / 16} P^{41 / 10+4 \delta+\varepsilon}$.

Proof. We may assume that the constants implied in defining $A_{i}$ and $B_{i}$ are the same for $i, \ldots, 4$. Then $A_{i}=I \backslash B_{i}, i=1, \ldots, 4$.

Let $A=A_{1} \cap A_{2} \cap A_{3} \cap A_{4}$ and

$$
\begin{aligned}
S= & \left(B_{1} \cap B_{2}\right) \cup\left(B_{1} \cap A_{2}\right) \cup\left(B_{2} \cap A_{1}\right) \cup\left(B_{3} \cap A_{1} \cap A_{2}\right) \\
& \cup\left(B_{4} \cap A_{1} \cap A_{2}\right) \cup \bigcup_{j=5}^{8}\left(B_{j} \cap A\right) .
\end{aligned}
$$

(i)

$$
\begin{aligned}
\int_{B_{1} \cap B_{2}}|V(\alpha)| d \alpha & \ll\left|\lambda_{1}\right|^{-1 / 4}\left|\lambda_{2}\right|^{-1 / 4} P^{3 / 2+4 \delta}\left(\prod_{i=3}^{4} \prod_{j=5}^{8} K_{i j}(2,4)\right)^{1 / 8} \\
& \ll \Pi^{-3 / 16} P^{41 / 10+4 \delta+\varepsilon} .
\end{aligned}
$$

By Lemma 2.7 and notice that $\left|\lambda_{1}\right|,\left|\lambda_{2}\right| \geqslant\left|\lambda_{j}\right|$ for $j=3,4, \ldots, 8$.

(ii)

$$
\begin{aligned}
& \int_{B_{1} \cap A_{2}}|V(\alpha)| d \alpha_{i} \\
& \ll\left|\lambda_{1}\right|^{-1 / 4} P^{3 / 4+2 \delta}\left(\prod_{j=5}^{8} \int_{A_{2}}\left|S_{2}(\alpha)\right|^{3}\left|S_{j}(\alpha)\right|^{4} d \alpha\right)^{1 / 12}\left(\prod_{i=3}^{4} \prod_{j=5}^{8} K_{i j}(3,4)\right)^{1 / 12} .
\end{aligned}
$$


By Lemma 2.9,

$$
\begin{aligned}
& \ll\left|\lambda_{1}\right|^{-1 / 4}\left|\lambda_{2}\right|^{-2 / 9}\left|\lambda_{3}\right|^{-7 / 36}\left|\lambda_{4}\right|^{-7 / 36}\left|\lambda_{5} \cdots \lambda_{8}\right|^{-19 / 108} P^{41 / 10+4 \delta+\varepsilon} \\
& \ll \Pi^{-3 / 16} P^{41 / 10+4 \delta+\varepsilon} .
\end{aligned}
$$

Similarly for $B_{2} \cap A_{1}$.

(iii)

$$
\begin{aligned}
& \int_{B_{3} \cap\left(A_{1} \cap A_{2}\right)}|V(\alpha)| d \alpha \\
& \ll\left|\lambda_{3}\right|^{-1 / 4} P^{3 / 4+2 \delta}\left(\prod_{\substack{k=1 \\
k \neq 3}}^{4} \prod_{j=5}^{8} \int_{A_{1} \cap A_{2}}\left|S_{k}(\alpha)\right|^{3}\left|S_{j}(\alpha)\right|^{4} d \alpha\right)^{1 / 12} .
\end{aligned}
$$

By Lemma 2.9,

$$
\begin{aligned}
& \ll\left|\lambda_{1}\right|^{-3 / 16}\left|\lambda_{2}\right|^{-2 / 9}\left|\lambda_{3}\right|^{-1 / 4}\left|\lambda_{4}\right|^{-7 / 36}\left|\lambda_{5} \cdots \lambda_{8}\right|^{-5 / 27} P^{41 / 10+4 \delta+\varepsilon} \\
& \ll \Pi^{-3 / 16} P^{41 / 10+4 \delta+\varepsilon} .
\end{aligned}
$$

Similarly for $B_{4} \cap A_{1} \cap A_{2}$.

(iv) For $j=5, \ldots, 8$,

$$
\begin{aligned}
\int_{B_{j} \cap A}|V(\alpha)| d \alpha & \ll\left|\lambda_{j}\right|^{-1 / 4} P^{3 / 5+2 \delta}\left(\prod_{k=1}^{4} \prod_{\substack{m=5 \\
m \neq j}}^{8} \int_{A_{k}}\left|S_{k}(\alpha)\right|^{4}\left|S_{m}(\alpha)\right|^{3} d \alpha\right)^{1 / 12} \\
& \ll\left|\lambda_{j}\right|^{-1 / 4} P^{3 / 5+2 \delta}\left(\prod_{k=1}^{4} \prod_{\substack{m=5 \\
m \neq j}}^{8} K_{k, m}(2,6) \int_{A_{k}}\left|S_{k}(\alpha)\right|^{6} d \alpha\right)^{1 / 24} .
\end{aligned}
$$

By Lemmas 2.7, 2.8 and using the fact that $P>\Pi$,

$$
\ll \Pi^{-1 / 6} P^{4+2 \delta} \ll \Pi^{-3 / 16} P^{41 / 10+4 \delta+\varepsilon} .
$$

Summing up (i) to (iv) the desired result follows.

4. Major arcs for the proof of Theorem 1. We define

$$
\begin{aligned}
M=\left\{\alpha \in S: S_{i}(\alpha)\right. & \gg\left|\lambda_{i}\right|^{-1 / 4} P^{3 / 4+2 \delta}, i=1, \ldots, 4, \\
& \text { and } \left.S_{j}(\alpha) \gg\left|\lambda_{j}\right|^{-1 / 4} P^{3 / 5+2 \delta}, j=5, \ldots, 8\right\} .
\end{aligned}
$$

Since we have assumed the constants implied are greater than or equal to 1 , by Lemma 2.4, the rational approximation $a_{i} / q_{i}$ which satisfies (4) must satisfy

$$
q_{i}<\left(\left|\lambda_{i}\right|^{-1 / 3} P\right)^{1-\delta}, \quad i=1, \ldots, 4 ; \quad q_{j}<\left(\left|\lambda_{j}\right|^{-1 / 3} P^{4 / 5}\right)^{1-\delta}, j=5, \ldots, 8 .
$$

By Lemma 2.3, this will imply that

(11)

$$
\begin{array}{lll}
0<q_{i}<\left(\left|\lambda_{i}\right|^{-1 / 3} P\right)^{3 / 4}, & \left|\beta_{i} / \lambda_{i}\right|<\left|\lambda_{i}\right|^{-1 / 12} P^{-11 / 4-2 \delta}, & i=1, \ldots, 4, \\
0<q_{j}<\left(\left|\lambda_{j}\right|^{-1 / 3} P^{4 / 5}\right)^{3 / 4}, & \left|\beta_{j} / \lambda_{j}\right|<\left|\lambda_{j}\right|^{-1 / 12} P^{-11 / 5-2 \delta}, & j=5, \ldots, 8 .
\end{array}
$$

We consider several lemmas before we proceed. 
Lemma 4.1. (i) If $P>4\left|\lambda_{i}\right|^{1 / 2}$, then there is at most one approximation $a_{i} / q_{i}$ to $\lambda_{i} \alpha$ such that (4) and (11) hold.

(ii) If $P>4\left|\lambda_{j} \lambda_{k}\right|$ and (4) and (11) hold for $i=j, k(j \neq k)$, then

$$
a_{j} / \lambda_{j} q_{j}=a_{k} / \lambda_{k} q_{k}
$$

(iii) If (4) and (11) hold, then

$$
\begin{aligned}
& \left|\beta_{i}\right|<q_{i}^{-1}\left(\left|\lambda_{i}\right|^{-1 / 3} P\right)^{-2-\delta} \quad i=1, \ldots, 4, \\
& \left|\beta_{j}\right|<q_{j}^{-1}\left(\left|\lambda_{i}\right|^{-1 / 3} P^{4 / 5}\right)^{-2-\delta}, \quad j=5, \ldots, 8 .
\end{aligned}
$$

Proof. The proofs are essentially the same as $[9$, Lemma 8$]$. We prove (iii) only.

(iii)

$$
\begin{aligned}
\left|\beta_{i}\right| q_{i} & \leqslant\left|\lambda_{i}\right|\left|\lambda_{i}\right|^{-1 / 12} P^{-11 / 4-2 \delta}\left(\left|\lambda_{i}\right|^{-1 / 4} P^{3 / 4}\right) \quad(i=1, \ldots, 4) \\
& <\left(\left|\lambda_{i}\right|^{-1 / 3} P\right)^{-2-\delta} .
\end{aligned}
$$

Similarly for $j=5, \ldots, 8$.

Suppose that $\alpha \in M$ and for each $i$ let $a_{i} / q_{i}$ be an approximation which satisfies (4) and (11). Since we have assumed $P>\Pi^{35 / 24}$, by Lemma 4.1(i), $a_{i} / q_{i}$ are unique for $\lambda_{i} \alpha$ and, by (ii), $a_{j} / \lambda_{j} q_{j}=a_{k} / \lambda_{k} q_{k}$, for all $j, k$. Hence there exist unique integers $a, q$ such that $(a, q)=1, q>0$ and

$$
a_{i} / q_{i}=\lambda_{i} a / q
$$

for all $i$. Let

$$
\delta_{i}=\left(\lambda_{i}, q\right), \quad i=1, \ldots, 8
$$

Then

$$
a_{i}=\lambda_{i} a / \delta_{i}, \quad q_{i}=q / \delta_{i} .
$$

By (11), we have

$$
0<q \leqslant \min _{\substack{1 \leqslant i \leqslant 4 \\ 5 \leqslant j \leqslant 8}}\left\{\delta_{i}\left|\lambda_{i}\right|^{-1 / 4} P^{3 / 4}, \delta_{j}\left|\lambda_{j}\right|^{-1 / 4} P^{3 / 5}\right\}
$$

and

$$
|\alpha-a / q| \leqslant\left|\lambda_{1}\right|^{-1 / 12} P^{-11 / 4-2 \delta} .
$$

Since $P>\Pi^{35 / 24}$ and (6) is satisfied, this implies $P>\left|\lambda_{1}\right|^{35 / 24}$ and $P>\left|\lambda_{j}\right|^{35 / 8}$ for $j=3, \ldots, 8$.

$$
\begin{aligned}
P^{19 / 35} & \leqslant \min \left\{\left|P^{24 / 35}\right|^{-1 / 4} P^{3 / 4},\left(P^{8 / 35}\right)^{-1 / 4} P^{3 / 5}\right\} \\
& \leqslant \min _{\substack{1 \leqslant i \leqslant 4 \\
5 \leqslant j \leqslant 8}}\left\{\delta_{i}\left|\lambda_{i}\right|^{-1 / 4} P^{3 / 4}, \delta_{j}\left|\lambda_{j}\right|^{-1 / 4} P^{3 / 5}\right\} \\
& \leqslant\left(P^{8 / 35}\right)^{3 / 4} P^{3 / 5} \leqslant P^{4 / 5} .
\end{aligned}
$$

We define $I_{a, q}$ to be the interval determined by (16). By previous argument, if $\alpha \in M$, then every rational approximation $a_{i} / q_{i}$ satisfying (4) must satisfy (11), which implies $a \in I_{a, q}$ for some $a, q$ such that $0 \leqslant a \leqslant q-1$ and $q$ satisfies (15). 
Conversely, suppose that $a, q$ are integers such that $(a, q)=1, q>0,0 \leqslant a \leqslant q$ -1 and (15) holds, where the $\delta_{i}$ are defined by (13). If $\alpha$ belongs to the interval $I_{a, q}$ defined as above, then $\alpha \in I$ and the approximation $a_{i} / q_{i}$, such that $\left(a_{i}, q_{i}\right)=1$ and $q_{i}>1$, defined by (12), satisfy (11) and (14), and these intervals do not overlap because of the uniqueness of the $a_{i} / q_{i}$.

By Lemma 4.1(iii), we can always use Lemma 2.3 to estimate $S_{i}(\alpha)$ for $\alpha$ in $M$. We can now estimate the contribution from $M$ to $\Re(P)$.

LEMMA 4.2. If $\delta \leqslant 1 / 30$,

$$
\begin{aligned}
\int_{M} V(\alpha) d \alpha=\Pi^{-1 / 3} \Im R(P) & O\left(P^{39 / 10}\right) \\
\text { where } \subseteq & =\sum_{q=1}^{\infty} \sum_{\substack{a=0 \\
(a, q)=1}}^{q-1}\left(q_{1} \cdots q_{8}\right)^{-1} S\left(a_{1}, q_{1}\right), \ldots, S\left(a_{8}, q_{8}\right),
\end{aligned}
$$

the $a_{i}, q_{i}$ are defined by (13) and (14), and $R(P) \gg P^{21 / 5}$.

Proof. Suppose $\alpha \in M$ and define $a_{i} / q_{i}$ by (13) and (14) and write

$$
\beta=\alpha-a / q=\beta_{i} / \lambda_{i}, \quad i=1, \ldots, 8 .
$$

Then by Lemma 2.3,

$$
V(\alpha)=\Pi^{-1 / 3}\left(\prod_{i=1}^{4} q_{i}^{-1} S\left(a_{i}, q_{i}\right) I( \pm \beta)\right)\left(\prod_{j=5}^{8} q_{j}^{-1} S\left(a_{j}, q_{j}\right) I^{\prime}( \pm \beta)\right)+E,
$$

where \pm is the sign of $\lambda_{i}$ and the error $E$ satisfies

$$
\begin{aligned}
E & \ll \sum_{i=1}^{8} q_{i}^{2 / 3+\varepsilon}\left(\prod_{\substack{j=1 \\
j \neq i}}^{8}\left|\lambda_{j}\right|^{-1 / 3} q_{j}^{-1 / 3}\right) P^{32 / 5}\left(\min \left(1, P^{-3}|\beta|^{-1}\right)\right)^{2} \\
& \ll q^{-5 / 3+\varepsilon}\left(\min \left(1, P^{-3}|\beta|^{-1}\right)\right)^{2} .
\end{aligned}
$$

Since $\left|\lambda_{i}\right|^{-1} q \leqslant q_{i} \leqslant q$ for all $i$ by (13) and (14),

$$
\begin{aligned}
\int_{M} E d \alpha & \ll \sum_{q}^{\prime} \sum_{a}^{\prime} \int_{I_{a, q}} q^{-5 / 3+\varepsilon} P^{32 / 5} \min \left(1, P^{-6}|\beta|^{-2}\right) d \alpha \\
& \ll \sum_{q}^{\prime} \sum_{a}^{\prime} q^{-5 / 3+\varepsilon} P^{17 / 5} \ll P^{19 / 5} \quad \text { by }(17),
\end{aligned}
$$

where $\Sigma_{q}^{\prime} \Sigma_{a}^{\prime}$ denotes the range of $q$ defined in $(15),(a, q)=1$ and $0 \leqslant a<q$. Since the $I_{a, q}$ do not overlap, it follows that

$$
\begin{aligned}
\int_{M} V(\alpha) d \alpha= & \Pi^{-1 / 3} \sum_{q}^{\prime} \sum_{a}^{\prime}\left(q_{1} \cdots q_{8}\right)^{-1} S\left(a_{1}, q_{8}\right), \ldots, S\left(a_{8}, q_{8}\right) \\
& \times \int_{I_{a, q}} \prod_{i=1}^{4} I( \pm \beta) \prod_{j=5}^{8} I^{\prime}(\beta) d \alpha+O\left(P^{19 / 5}\right) .
\end{aligned}
$$


The error caused by replacing $I_{a, q}$ in (19) by the interval $|\alpha-a / q| \leqslant \frac{1}{2}$ is

$$
\ll \Pi^{-1 / 3} \sum_{q}^{\prime} \sum_{a}\left(q_{1} \cdots q_{8}\right)^{-1 / 3} \int_{I_{a . q}^{*}} P^{36 / 5} \min \left(1, P^{-3}|\beta|^{-1}\right)^{4} d \alpha,
$$

where $I_{a, q}^{*}$ is the set $\alpha=a / q+\beta$ such that

$$
\frac{1}{2} \geqslant|\beta|>\left|\lambda_{1}\right|^{-1 / 12} P^{-11 / 4-2 \delta} \geqslant P^{-17 / 6-2 \delta} \geqslant P^{-3},
$$

since $P>\left|\lambda_{1}\right|$ and $2 \delta \leqslant \frac{1}{6}$.

Now, for any pair $a, q$,

$$
\Pi^{-1 / 3}\left(q_{1} \cdots q_{8}\right)^{-1 / 3} P^{-24 / 5} \int_{I_{a, q}^{*}} \beta^{-4} d \alpha \ll q^{-8 / 3} P^{37 / 10+6 \delta} .
$$

Hence the total error is certainly

$$
\ll P^{37 / 10+6 \delta} \sum_{q=1}^{\infty} \sum_{a=0}^{q-1} q^{-8 / 3} \ll P^{39 / 10}
$$

provided that $6 \delta \leqslant \frac{1}{5}$. Thus we have

$$
\begin{aligned}
\int_{M} V(\alpha) d \alpha= & \Pi^{-1 / 3} \sum_{q}^{\prime} \sum_{a}^{\prime}\left(q_{1} \cdots q_{8}\right)^{-1} S\left(a_{1}, q_{1}\right) \cdots S\left(a_{8}, q_{8}\right) R(P) \\
& +O\left(P^{19 / 5}\right)
\end{aligned}
$$

where

$$
R(P)=\int_{-1 / 2}^{1 / 2} \prod_{i=1}^{4} I(\beta) \prod_{j=5}^{8} I^{\prime}(\beta) d \beta=3^{-8} \sum_{m_{1}, \ldots, m_{8}}\left(m_{1} \cdots m_{8}\right)^{-2 / 3}
$$

by definition of $I(\beta)$ and $I^{\prime}(\beta)$, where

$$
\begin{gathered}
P^{3} \leqslant m_{i} \leqslant 8 P^{3}, \quad i=1, \ldots, 4, \\
P^{12 / 5} \leqslant m_{j} \leqslant 8 P^{12 / 5}, \quad j=5, \ldots, 8,
\end{gathered}
$$

and $\pm m_{1} \pm m_{2} \pm \cdots \pm m_{8}=0$.

Since either $\lambda_{2}$ or $\lambda_{3}$ has sign different then $\lambda_{1}$, without loss of generality, we assume $\lambda_{2} \lambda_{1}<0$. So now the last condition is

$$
m_{1}=m_{2} \mp m_{3} \mp m_{4} \mp \ldots \mp m_{8}
$$

for each $\left(m_{2}, \ldots, m_{4}\right)$ such that

$$
4 P^{3} \leqslant m_{2} \leqslant 5 P^{3}, \quad P^{3} \leqslant m_{i} \leqslant 5 P^{3} / 4, \quad i=3,4,
$$

and the integer $m_{1}$ defined by (23) satisfies $P^{3} \leqslant m_{1} \leqslant 8 P^{3}$. Thus the number of solutions of (23) such that (22) holds is $\gg P^{9} P^{48 / 5}$. For any such solution we have

$$
\left(m_{1} \cdots m_{8}\right)^{-2 / 3} \geqslant 2^{-16} P^{-8} P^{-32 / 5} \text {. }
$$

Thus $R(P) \gg P^{21 / 5}$ as required. 
The error caused by extending the range of summation for $q$ to infinity is

$$
\begin{aligned}
& \ll \Pi^{-1 / 3} \sum_{q} \sum_{a}\left(q_{1} \cdots q_{8}\right)^{-1 / 3} P^{36 / 5} \int_{-1 / 2}^{1 / 2} \min \left(1, P^{-6}|\beta|^{-2}\right) d \beta \\
& \ll \Pi^{-1 / 3} \sum_{q} \sum_{a}\left(q_{1} \cdots q_{8}\right)^{-1 / 3} P^{21 / 5},
\end{aligned}
$$

where $q$ is summed over the range $q>P^{19 / 35}$, and $a$ is as before. We consider a particular set of divisors $\delta_{1}, \ldots, \delta_{8}$ of $\lambda_{1}, \ldots, \lambda_{8}$, repectively. The contribution to the above from those pairs $a, q$ corresponding to $\delta_{1}, \ldots, \delta_{8}$ is

$$
\ll \Pi^{-1 / 3}\left(\delta_{1} \cdots \delta_{8}\right)^{1 / 3} P^{21 / 5} \sum_{q>P^{19 / 35}} q^{-5 / 3} .
$$

Thus the contribution from these $a, q$ is

$$
\ll \Pi^{-1 / 3} P^{21 / 5}\left(\delta_{1} \cdots \delta_{8}\right)^{1 / 3} P^{-38 / 105} \ll \Pi^{-1 / 3} P^{21 / 5} \Pi^{1 / 3} P^{-38 / 105} \ll P^{39 / 10-\varepsilon} .
$$

Since $\Pi \ll P$, the number of different possibilities for $\delta_{1} \cdots \delta_{8}$ is $O\left(P^{\varepsilon}\right)$, and it follows that the total error is $\ll P^{39 / 10}$. Hence the result followed.

5. The singular series. Firstly, we assume that the $\lambda_{i}$ are cube-free and no prime divides more than five of them. The following lemma shows that the assumption may be made without loss of generality.

LEMMA 5.1. If Theorem 1 holds provided the $\lambda_{i}$ are cube-free nonzero integers such that no prime divides more than five of them, then it holds for every set of nonzero integral $\lambda_{i}$.

Proof. The proof is essentially the same as [9, Lemma 5].

We now investigate the singular series $\subseteq$ defined by (18). Since $S\left(\lambda_{i} a, q\right)=$ $\delta_{i} S\left(a_{i}, q_{i}\right)$ and $S\left(\lambda_{i} a, q\right)$ is periodic of period $q$, we may rewrite the series as

$$
\sum_{q=1}^{\infty} \sum_{\substack{a=1 \\(a, q)=1}}^{q} q^{-8} S\left(\lambda_{1} a, q\right) \cdots S\left(\lambda_{8} a, q\right) .
$$

For $p$ prime, we define

$$
\chi(p)=1+\sum_{\nu=1}^{\infty} \sum_{a=1}^{p^{\nu}} P^{-8 \nu} \prod_{i=1}^{8} S\left(\lambda_{i} a, P^{\nu}\right)
$$

and $M\left(P^{\nu}\right)$ to be the number of solutions $\left(\bmod p^{\nu}\right)$ of the congruence

$$
\sum_{i=1}^{8} \lambda_{i} x_{i}^{3} \equiv 0 \quad\left(\bmod P^{\nu}\right)
$$

Similar to $[9, \S 6]$, we have

$$
\chi(p)=\lim _{\nu \rightarrow \infty} P^{-7 \nu} M\left(P^{\nu}\right) \text { and } \subseteq=\prod_{P} \chi(p),
$$

where the product is over all primes $p$. 
LEMMA 5.2. (i) If $s \geqslant 3, p$ is any prime, and $p \nmid\left|\lambda_{1} \cdots \lambda_{s}\right|$, then there is a nontrivial solution (i.e. one with some $x_{i} \neq 0$ ) of

$$
\sum_{i=1}^{s} \lambda_{i} x_{i}^{3} \equiv 0 \quad(\bmod p) .
$$

(ii) Suppose that $p \neq 3$ is a prime such that $\lambda_{1}, \ldots, \lambda_{s}$ are not divisible by $p$ and $\lambda_{s+1}, \ldots, \lambda_{8}$ are divisible by $p$, and let $\Re(p)$ denote the number of nontrivial solutions of (27). Then for all $\nu>0$,

$$
M\left(p^{\nu}\right) \geqslant p^{7(\nu-1)+(8-s)} \Re(p) .
$$

(iii) If $\lambda_{1}, \ldots, \lambda_{8}$ are cube-free and $\nu \geqslant 6$, then $M\left(3^{\nu}\right) \geqslant 3^{7(\nu-6)}$.

Proof. The proof is essentially the same as [9, Lemma 10].

LEMMA 5.3. (i) There is an absolute constant $C_{1}>0$ such that

$$
\prod_{p} \chi(p) \geqslant \frac{1}{2} \quad\left(p \nmid \Pi, p>C_{1}\right) .
$$

(ii) There is a constant $C_{2}=C_{2}(\varepsilon)>0$ such that

$$
\prod_{p} \chi(p) \geqslant \Pi^{-\varepsilon} \quad\left(p \nmid \Pi, p>C_{2}\right) .
$$

(iii) We have $\subseteq \gg \Pi^{-\varepsilon}$.

(The products in (i) and (ii) are over all primes $p$ which satisfy the condition in parentheses.)

Proof. See [9, Lemma 11].

6. Completion of the proof of Theorem 1. Assuming that $P>\Pi^{35 / 24}$ and $\delta \leqslant \frac{1}{30}$, by Lemmas 4.2 (with $\varepsilon=\delta$ ), 5.2 and 3.1 (since we have $I \backslash M \subset S$ ), we have

$$
\Re(P)=C_{\varepsilon} \Pi^{-(1 / 3)-\varepsilon} P^{21 / 5}+E,
$$

where $C_{\varepsilon}>0$ and

$$
\begin{aligned}
E & \ll \Pi^{-3 / 16} P^{41 / 10+5 \delta}+P^{39 / 10} \ll \Pi^{-3 / 16} P^{41 / 10+5 \delta} \\
& \ll \Pi^{-1 / 3} P^{21 / 5}\left(\Pi^{-35 / 24} P^{1-\theta}\right)^{-1 / 10} P^{5 \delta-\delta / 10} .
\end{aligned}
$$

For given $\theta>0$, we choose $\varepsilon>0$ and $\delta>0$ such that $\varepsilon+5 \delta \leqslant \theta / 20, \delta<1 / 30$. Then $P^{5 \delta-\theta / 10} \leqslant P^{-\varepsilon-\theta / 20}$.

Since $P>\Pi^{35 / 24}$ certainly holds if $P^{1-\theta}>\Pi^{35 / 24}$, it now follows from the preceding paragraph that there is a constant $D_{\theta}$ such that $\Re(P)>0$ provided $P^{1-\theta}>D_{\theta} \Pi^{35 / 24}$. By the remarks at the beginning of $\S 3$ this completes the proof of Theorem 1 .

\section{REFERENCES}

1. H. Davenport, On Waring's problem for cubes, Acta Math. 71 (1939), 123-143.

2. In_efinite quadratic forms in many variables, Mathematika 3 (1956), 81-101.

3. _ Cubic forms in 32 variables, Philos. Trans. Roy. Soc. London Ser. A 251 (1959), 193-232.

4. Analytic methods for Diophantine equations and Diophantine inequalities, Ann Arbor Publ., Ann Arbor, Mich., 1963. 
5. H. Davenport and H. Heilbronn, On indefinite quadratic forms in five variables, J. London Math Soc. 21 (1946), 185-193.

6. H. Davenport and K. F. Roth, The solubility of certain Diophantine inequalities, Mathematika 2 (1955), 81-96.

7. I. Danicic, The solubility of certain Diophantine inequalities, Proc. London Math. Soc. (3) 8 (1958), $161-176$.

8. D. J. Lewis, Cubic congruences, Michigan Math. J. 4 (1957), 85-95.

9. J. Pitman and D. Ridout, Diagonal cubic equations and inequalities, Proc. Roy. Soc. Ser. A 297 (1967), 476-502.

10. T. Nagell, Introduction to number theory, Wiley, New York, 1951

Department of Mathematics, University of Hong Kong, Hong Kong

Current address: Department of Mathematics, Stanford University, Stanford, California 94305 